\title{
Auto-interruptions et disfluences en français parlé dans quatre corpus du CID
}

Auto-interruptions and disfluencies in spoken French: four corpus of the CID

(Corpus of Interactional Data)

\section{Berthille Pallaud, Stéphane Rauzy et Philippe Blache}

\section{(2) OpenEdition}

Édition électronique

URL : https://journals.openedition.org/tipa/995

DOI : 10.4000/tipa.995

ISSN : 2264-7082

Éditeur

Laboratoire Parole et Langage

Référence électronique

Berthille Pallaud, Stéphane Rauzy et Philippe Blache, « Auto-interruptions et disfluences en français parlé dans quatre corpus du CID », TIPA. Travaux interdisciplinaires sur la parole et le langage [En ligne], 29 | 2013, mis en ligne le 19 décembre 2013, consulté le 10 mars 2023. URL : http:// journals.openedition.org/tipa/995; DOI : https://doi.org/10.4000/tipa.995

Ce document a été généré automatiquement le 10 mars 2023.

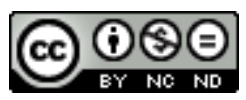

Creative Commons - Attribution - Pas d'Utilisation Commerciale - Pas de Modification 4.0 International - CC BY-NC-ND 4.0

https://creativecommons.org/licenses/by-nc-nd/4.0/ 


\title{
Auto-interruptions et disfluences en français parlé dans quatre corpus $d u$ CID $^{1}$
}

\author{
Auto-interruptions and disfluencies in spoken French: four corpus of the CID \\ (Corpus of Interactional Data)
}

Berthille Pallaud, Stéphane Rauzy et Philippe Blache

\section{Introduction}

1 Plusieurs phénomènes qualifiés de disfluents peuvent être observés dans les énoncés oraux de parole spontanée ${ }^{2}$. Ils concernent aussi bien les niveaux phonétique acoustique et prosodique (Cole et al. 2005 ; Shriberg, 1995 et 1999; Yoonsook et al., 2005 ; Shu Chuan Tseng, 1999 ; Schuller et al. 2008) que le niveau morphosyntaxique qui fera l'objet de la présente étude (Dister, 2008). Les variations dans le rythme de la fluence verbale (Pasdeloup, 1992 ; Duez, 2007) peuvent aller jusqu'à l'auto-interruption de mots ou de syntagmes. Ces auto-interruptions et auto-variations dans la fluence verbale sont signalées, dans la grande majorité des cas (mais pas dans tous, comme on le verra), par l'apparition d'une ou de plusieurs sortes d'événements: pauses silencieuses ou remplies, items insérés au milieu d'un syntagme ou même d'un mot. Elles peuvent s'accompagner de perturbations qui se traduisent ${ }^{3}$, en particulier, par des effets sur l'organisation morphosyntaxique du flux verbal, les plus fréquemment cités étant les reprises d'énoncés, les auto-réparations et les inachèvements de syntagme ou de mots (Clark \& Wasow, 1998 ; Henry \& Pallaud, 2003 ; Guénot, 2005 ; Pallaud \& Henry, 2006 et 2007$)^{4}$ :

YM_1506 un gosse j'en // j'en // moi j'en voulais pas YM_1739 ça commençait fé- // en février ou un truc comme ça

YM_1977 moi je // quand il est parti il nous a laissé tous ses // euh tous ses épices 
2 En effet, aucun locuteur standard ne parle, de façon non préparée, sans produire ces phénomènes qui sont propres à la parole spontanée et n'ont donc rien d'un dysfonctionnement. Les productions disfluentes varient d'un locuteur à l'autre en quantité mais elles sont toujours présentes à l'oral (Blanche-Benveniste, 1997 ; Cutler, 1981 ; Den, 2001 ; Fox Tree, 1995 ; Henry \& Pallaud, 2003d ; Jeanjean, 1984 ; Levelt, 1989 ; Pallaud, 2006).

3 Clark \& Wasow (1998), ainsi que Shriberg (1999) ont décrit la structure sous-jacente à ces perturbations: le Reparandum, lieu de la production source cessée au point de rupture, suivi de l'Interregnum (moment potentiellement utilisé par le locuteur) et enfin $\mathrm{du}$ Reparans $^{5}$ (lui aussi potentiel) qui correspond à la reprise du déroulement syntagmatique. L'avantage de cette description est qu'elle permet de distinguer ce qui précède l'interruption, ce qui lui succède éventuellement sans renouer le fil du discours et ce qui vient réparer, poursuivre ou laisser inachevée la construction syntaxique interrompue.

$4 \quad$ Notre identification des interruptions dans le flux verbal repose sur la détection de ce qui suit l'instant interruptif c'est-à-dire sur l'observation de l'espace Interregnum. Nous proposons donc de ne pas confondre ce qui se passe dans cet espace avec les effets morphosyntaxiques sur l'énoncé qui suit (le Reparans). Cela conduit à distinguer :

\section{$5 \quad \mathbf{1}^{*}$ Les indices d'interruption}

\section{2* Les disfluences lexicalisées}

$6 \quad 1^{*}$ Les indices d'interruption sont insérés dans l'énoncé et ont en commun de ne pas avoir de fonction syntaxique. La plupart des travaux consacrés à la disfluence ne distinguent pas les indices d'interruption (pauses remplies ou non, marqueurs de discours, incidentes parenthétiques) des effets de ces interruptions (par exemple Constant \& Dister, 2010 ; Meteer, 1995 ; Zelner, 1992) et se sont attachés à décrire les phénomènes acoustiques, phonétiques et prosodiques présents :

- au moment de l'interruption de l'énoncé (déformation de phonèmes, par exemple Shriberg, 1999)

- ou dans le moment qui suit cette interruption avant que l'énoncé ne reprenne ou soit définitivement interrompu (pauses silencieuses ou remplies, Duez, 2001, 2001b et les particules discursives, par exemple).

2* D'autres études (Pallaud \& Henry, 2004 ; Dister, 2007 et 2008) ont observé les effets énonciatifs des auto-interruptions dans des énoncés non préparés; les disfluences constituant une partie des effets de ces auto-interruptions involontaires du flux verbal dans le déroulement syntagmatique :

- reprise avec ou sans modification d'une partie de l'énoncé qui suit l'interruption.

• inachèvement de l'énoncé (mot ou syntagme).

Nous avons entrepris une étude des interruptions observables dans les énoncés de parole non préparée et de leurs effets morphosyntaxiques lors des séquences verbales qui leur succèdent. Si toutes les interruptions ne sont pas suivies d'une réorganisation morphosyntaxique un certain nombre sont perturbatrices.

9 Plutôt que de chercher à quantifier et analyser quelques types de disfluence préalablement définis comme les amorces de mots ou les répétitions, par exemple, (Adda-Decker et al., 2003; Bear et al., 1992; Boula de Mareüil et al., 2005), l'option choisie a été d'identifier toutes les auto-interruptions dans les énoncés et de décrire les conséquences morphosyntaxiques de ces ruptures dans le flux verbal. On verra, à cette 
occasion, que certaines auto-interruptions ne sont signalées que par ces perturbations morphosyntaxiques. Cette approche permet d'une part d'identifier de façon exhaustive toutes les auto-interruptions dans un énoncé et d'autre part de décrire, pour cette étude, les relations existant entre ces auto-interruptions et leurs effets morphosyntaxiques. Elle permettra notamment de préciser la notion de disfluence.

\section{Objectifs}

Notre approche propose de détecter toutes les auto-interruptions dans les énoncés (c'est-à-dire tous les indices d'interruption) et de décrire les effets morphosyntaxiques des interruptions dans le flux lexical (qu'ils soient, perturbateurs ou non) produits par le locuteur dans son énoncé. L'absence d'indices d'interruption ${ }^{6}$ ne garantit cependant pas toujours une fluence verbale puisque certaines interruptions sont observées seulement aux niveaux syntaxiques ou prosodiques. L'identification de l'interruption repose alors entièrement sur l'effet morphosyntaxique constaté : interruptions de mots ou de syntagmes définitives ou non, répétitions partielles ou complètes de mots ou de syntagmes.

\section{Méthode d'identification : le système d'annotation des auto-interruptions et des disfluences morphosyntaxiques}

11 La méthode d'identification et le système d'annotation des interruptions et des disfluences morphosyntaxiques ont été élaborés et appliqués sur deux dilogues du corpus du CID ( $\mathrm{AB}_{-} \mathrm{CM}$ et $\mathrm{AG}_{-} \mathrm{YM}$ ). Ce sont des corpus de paroles non préparées recueillis lors de conversations d'une heure entre deux locuteurs sur un thème proposé (Bertrand et al, 2009). Les locuteurs diffèrent quant au nombre de mots prononcés et diffèrent également quelque peu sur le temps de parole occupé.

Notre étude présente, d'une part, la méthode d'identification des interruptions qui repose majoritairement sur la détection des indices d'interruption et d'autre part le système d'annotation élaboré pour rendre compte de la variété des phénomènes d'interruption morphosyntaxique.

\subsection{La méthode d'identification des auto-interruptions et disfluences morphosyntaxiques}

13 Afin de décrire la totalité des interruptions dans les énoncés (en moyenne 1060 interruptions par locuteur) et la totalité des interruptions provoquant des perturbations morphosyntaxiques (en moyenne 492 disfluences par locuteur), nous avons employé successivement deux méthodes de détection, l'une semi-automatique et l'autre manuelle. Les deux méthodes utilisent le logiciel Praat comme outil d'identification, d'annotation et de description.

Méthode semi-automatique : il s'agit de localiser les interruptions du flux verbal grâce aux indices d'interruption afin de procéder à l'annotation du phénomène qui leur est lié. Les indices d'interruption sont constitués par des événements isolés (par exemple, une pause silencieuse) ou par plusieurs éléments se succédant et dit 
" associés » (par exemple, une pause remplie suivie d'un marqueur de discours ou d'une interjection). Ces indices d'interruption ont en commun de ne pas avoir de fonction syntaxique

AB_571 et euh \# et je euh bon moi j'étais dans un état un peu pas très bien

AB_620 C'était euh tu vois complètement loufoque comme si- ouais euh comme situation

Suivant ce que contiennent les espaces Interregnum, ces indices d'interruption peuvent être regroupés de la façon suivante :

- Ceux qui contiennent des pauses silencieuses (sp) et des pauses remplies (fp), seules ou associées à des éléments discursifs : ils signalent $64 \%$ des interruptions.

- Les interjections et les éléments discursifs succédant seuls aux interruptions ne représentent que $\mathbf{1 7} \%$ des cas ; une liste établie permettrait leur recherche automatisée ${ }^{7}$.

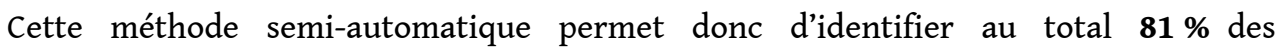
interruptions dans la fluence verbale (Fig.1).

Méthode manuelle : Il reste que certaines interruptions dans la fluence verbale (19\%; Fig.1) ne sont signalées que par leurs effets morphosyntaxiques. On n'observe aucun des éléments décrits précédemment; l'espace potentiel entre l'énoncé interrompu et celui qui lui succède (espace Interregnum) est vide. Quelques éléments discursifs comme les insertions parenthétiques qui interrompent un énoncé en provoquant souvent des réorganisations morphosyntaxiques ne peuvent pas non plus être détectée automatiquement $^{8}$. La méthode manuelle consiste donc à faire une lecture-écoute sémantique des transcriptions qui, à l'aide de paramètres prosodiques, sémantiques et/ ou syntaxiques, révèle ces ruptures.

AB_2187 ce devait être une boutique style mercerie je sais pas trop qui qui euh CM_2031 et y avait donc on était aux Etats Unis y avait des chaînes cryptées

Figure 1. Proportions des espaces Interregnum remplis ou non

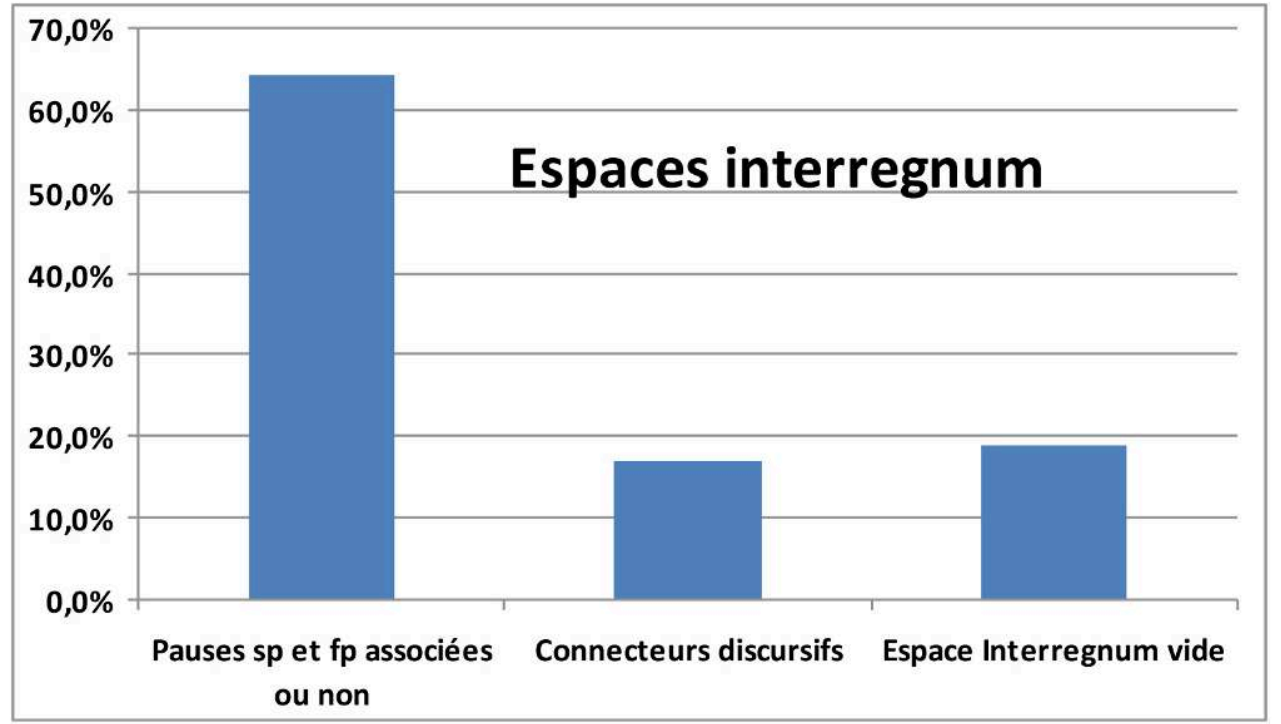


Qu'un cinquième des interruptions ne soient signalées que par une discordance syntaxique constitue pour notre méthode une difficulté certaine. Les locuteurs varient quant au nombre d'interruptions dans leurs énoncés mais la moyenne est supérieure à un millier ce qui conduit à identifier, pour chaque locuteur, environ deux cents disfluences non suivies d'un espace Interregnum détectable.

Une solution serait le repérage des discordances syntaxiques ce qui pourrait constituer une méthode de détection automatique de perturbations dans l'énoncé. Elle serait à évaluer par une confrontation avec les résultats de la méthode manuelle décrite ici.

\subsection{Le système d'annotation}

C'est autour du point d'interruption dans la fluence verbale que l'annotation du phénomène provoqué par cette rupture prend place. L'interruption peut se produire au milieu d'un mot (word truncation) ou au milieu d'un syntagme (phrase truncation) et délimite trois espaces formels dont la chronologie est la suivante: le Reparandum, l'espace Interregnum (Break point) et le Reparans. Chacun de ces espaces est défini par l'étendue des items (verbaux ou non).

$21 \mathbf{1}^{\circ}$ le Reparandum ( $\mathbf{R}$ ou $\left.\mathbf{I}\right)$ est ce qui, avant le point de rupture, contient une perturbation (fragment de mot ou de syntagme) et sera simplement poursuivi, repris, répété, modifié (R) ou abandonné (I), lors du Reparans.

$2^{\circ}$ l'Interregnum (Break interval B) est un moment potentiel avant que n'intervienne le Reparans. Il peut être vide ou contenir des indices de disfluence le plus souvent non lexicalisés (pauses remplies ou silencieuses, répétition de troncation, éléments discursifs ou parenthétiques plus ou moins longs, onomatopées, etc.)

$3^{\circ}$ le Reparans, partie potentielle de l'énoncé prononcé qui peut poursuivre, répéter ou modifier ce qui a été dit lors du Reparandum. Cet élément comporte deux situations selon qu'il est vide ou rempli.

$3.1^{\circ}$ Reparans vide : énoncé laissé inachevé ; il n'y a pas de Reparans codé.

$3.2^{\circ}$ Reparans non vide : Reparans (RA) qui comporte trois possibilités :

* une simple complétude du syntagme commencé et interrompu ${ }^{9}$

* la reprise partielle de l'énoncé déjà prononcé ce qui correspond à un entassement paradigmatique (le piétinement syntaxique selon Claire Blanche-Benveniste, 1997).

* la reprise de l'énoncé prononcé comporte des modifications.

Exemple :

tu perds un peu comment dire euh + un peu des repères

tu perds un peu (Reparandum)

comment dire euh + (Interregnum)

un peu (Reparans) des repères

L'analyse morphosyntaxique de ces disfluences lexicalisées (Pallaud 2002; Pallaud \& Henry, 2004) a montré que le Reparandum ne peut être identifié qu'à l'aide des éléments qui vont lui succéder et tout particulièrement ce qui va être repris de l'énoncé avant le point de rupture (c'est-à-dire le Reparans). Le nombre d'éléments contenus dans le Reparandum est déterminé par ce qui constitue le Reparans. Lorsqu'il y a inachèvement de l'énoncé le Reparandum est l'item tronqué ou le dernier item du syntagme laissé inachevé. 
L'annotation des disfluences présentes dans les corpus du CID rend compte de cette structure. Elle est faite sous Praat et portée dans le fichier TextGrid_Nom_ortho token où le son est aligné sur les éléments mots, articles, pronoms, etc. Elle est inscrite sur une ou plusieurs Tier (terminologie de Praat) selon que les disfluences sont ou non enchâssées. Celles-ci sont alignées sur les token concernés par les trois sortes d'éléments de la structure: dans l'ordre chronologique, le Reparandum, l'espace Interregnum et le Reparans. Il s'agit donc de définir les intervalles correspondant aux trois axes de la structure. Chaque élément de cette structure comporte, à son tour, plusieurs sortes d'informations qui vont être décrites et codées.

24 L'annotation peut donc se limiter à identifier les trois éléments de la structure (Reparandum, Interregnum et Reparans) ou décrire également chacun des types d'éléments qui les composent et dont la description peut se résumer par le tableau suivant (Blache et al. 2010).

Tableau 1. Système d'annotation des interruptions dans la fluence verbale

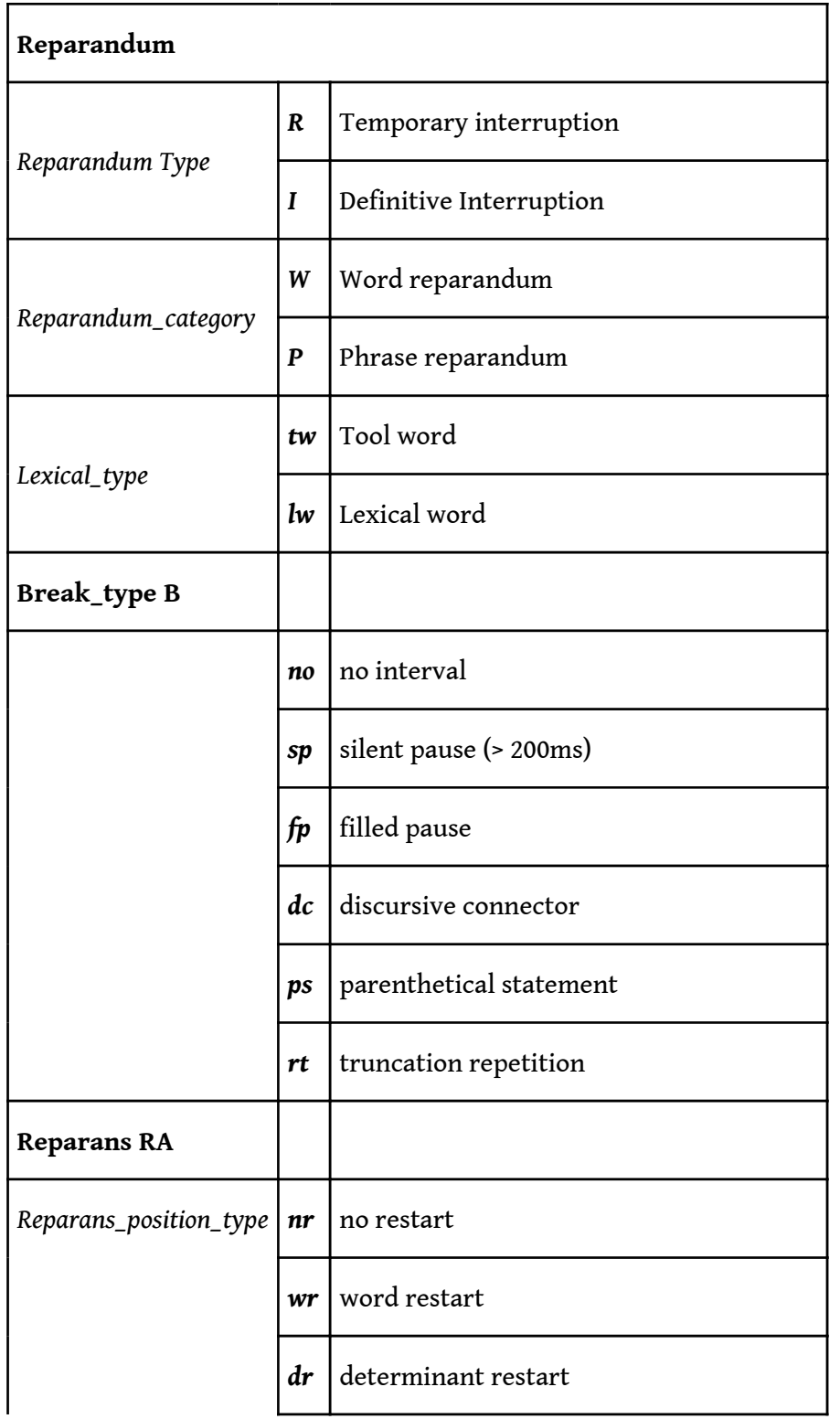




\begin{tabular}{|c|c|c|}
\hline & pr & phrase restart \\
\hline & or & other restart \\
\hline \multirow{5}{*}{ Reparans_type } & co & continuing the item \\
\hline & $w c$ & repairing without change \\
\hline & $r p$ & Repairing through repeating \\
\hline & $r c$ & repair with change in the truncated word \\
\hline & $\mathbf{r m}$ & repair with multiple change \\
\hline
\end{tabular}

\section{Quelques résultats (dilogues du CID : AG_YM et AB_CM)}

\subsection{Le nombre moyen de mots sans interruption}

Les interruptions varient en fonction des dilogues et des locuteurs. Leur fréquence (tableau 2) varie selon les locuteurs et représente en moyenne une rupture tous les 7,4 mots (de 6,2 à 9,8 mots selon les locuteurs). Plus le nombre de mots entre deux interruptions est faible, plus la fréquence de ces interruptions est forte.

Notre étude est centrée sur les effets morphosyntaxiques de l'interruption. Les interruptions du flux verbal quelles qu'elles soient ont divers effets possibles, au niveau prosodique et phonétique (de plus en plus étudiés; Shriberg et son équipe) mais aussi sur la séquence verbale qui suit. Elles ne sont pas toutes sources de disfluence c'est-àdire de réparation ou d'abandon et peuvent être sans effet sur la poursuite de l'énoncé (interruption simplement suspensive; voir plus loin). Trois effets de ces interruptions sont observés et étudiés :

$1^{*}$ L'énoncé est laissé inachevé (cas de Reparandum I) :

AB_2864 j'étais allée à un spectacle euh + au théâtre à la Rochelle \# avec // euh je me rappelle plus c'était super intéressant en fait c'était une violoniste connue

$2^{*}$ Poursuite après reprise d'une partie de l'énoncé déjà prononcé (cas RA avec reprise : sous catégories, $\mathbf{w r} ; \mathrm{dr}, \mathrm{pr}, \mathrm{or})$ :

YM_1640 mais de toutes façons tu es// euh tu es obligé

YM_1379 non non j'é-//j'écoute pas la radio

YM_1124 chaque fois tu ma-//tu devais marquer

YM_176 et puis je le //des fois je le faisais en rêve

Les cas d'abandon et de reprises sont considérés comme des interruptions disfluentes. Les cas de reprises comportent toujours au moins une répétition ce qui permet de les détecter automatiquement (Bigi \& Bertrand, 2012 sur les hétéro-répétitions). Après un moment temporairement elliptique, ces reprises sont fonctionnellement réparatrices 
(et souvent nommées « réparations »). Les interruptions qui laissent l'énoncé inachevé peuvent également être considérées comme des énoncés elliptiques mais définitives (Pallaud, 2008).

Toutefois ces deux types d'interruptions ne doivent pas être confondus avec des procédés rhétoriques (comme la répétition emphatique, par exemple) qui, eux, ne signalent pas une erreur dans l'élaboration de l'énoncé mais plutôt un effet de style (Pallaud, 2008).

313 3*Poursuite simple de l'énoncé (cas de Reparans RA,nr,co) :

YM_2432 tu le fais avec euh ce que tu veux

Nous avons nommé cette dernière catégorie d'interruptions «interruptions suspensives » puisqu'elles ne provoquent qu'une suspension (elle aussi temporaire) et non une réorganisation de l'énoncé.

Les interruptions disfluentes comme celles qui ne sont que suspensives ont, en moyenne, la même fréquence (respectivement une toutes les 15,4 mots et une toutes les 14,4 mots ; tableau 2). Un peu moins de la moitié des interruptions sont disfluentes. Les locuteurs diffèrent fortement quant aux interruptions disfluentes mais moins que pour les simples suspensions du flux verbal; si le locuteur YM a une fréquence de disfluences élevées (une tous les 12,3 mots), chez la locutrice AB cette disfluence est plus de deux fois moins élevée (une tous les 27,3 mots).

Tableau 2. Fréquence (en nombre moyen de mots entre deux interruptions ;) des diverses interruptions dans les énoncés des quatre locuteurs

\begin{tabular}{|l|l|l|l|}
\hline $\begin{array}{l}\text { Une interruption tous les } \\
\text { mots }\end{array}$ & $\begin{array}{l}\text { Toute } \\
\text { interruption }\end{array}$ & $\begin{array}{l}\text { Interruption } \\
\text { suspensive }\end{array}$ & $\begin{array}{l}\text { Interruption } \\
\text { disfluente }\end{array}$ \\
\hline Locuteur YM & 6,2 & 12,6 & 12,3 \\
\hline Locuteur AG & 8,5 & 14,7 & 13,6 \\
\hline Locuteur AB & 9,8 & 15,5 & 26,5 \\
\hline Locuteur CM & 7,9 & 15,6 & 16,3 \\
\hline Moyenne & 7,4 & 15,4 & 14,4 \\
\hline
\end{tabular}

\section{Les enchâssements d'interruptions dans les énoncés}

La méthode ayant consisté à identifier et annoter toutes les interruptions survenant dans les énoncés, et certaines se chevauchant, l'annotateur doit utliser plusieurs Tiers pour rendre compte de ces chevauchements. Le Reparans, en partie ou en totalité peut être à son tour Reparandum de sorte qu'il est nécessaire d'ouvrir une autre tier pour annoter cette nouvelle interruption disfluente (Tableau 3).

Tableau 3. Exemple d'enchâssement ; Locuteur CM_2350

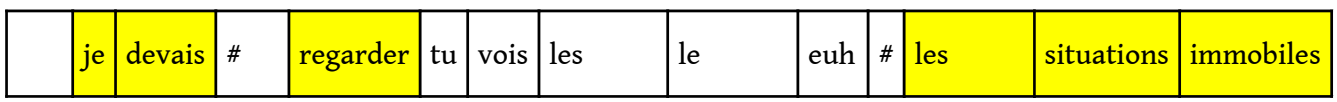




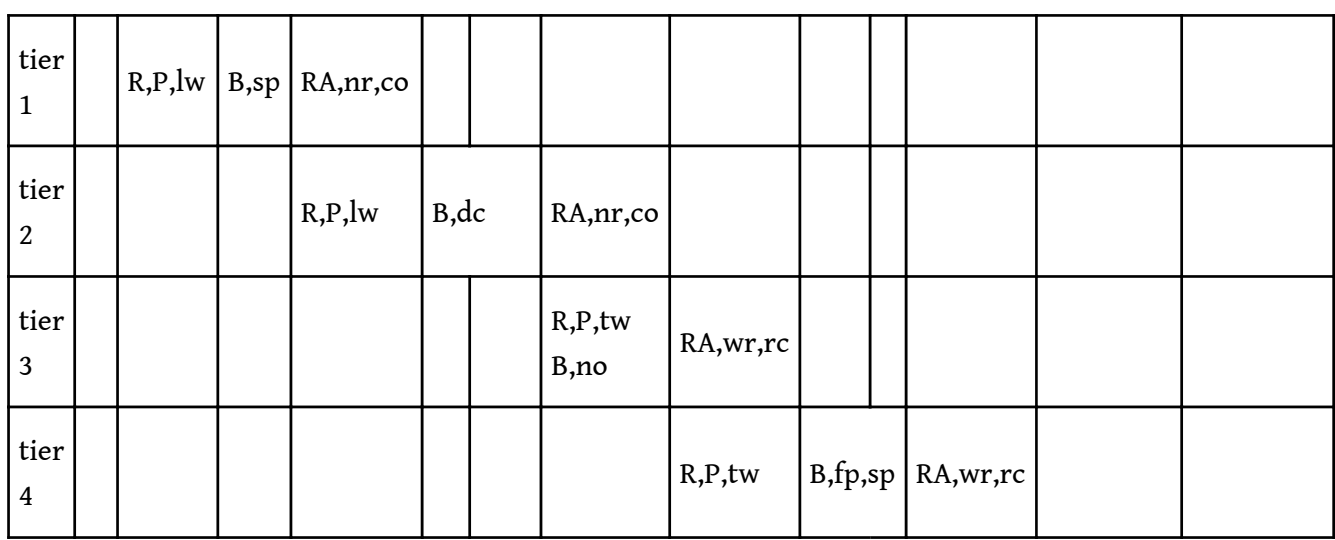
Dans notre étude, les pourcentages de ces chevauchements varient d'un locuteur à l'autre et sont compris entre 21 et $30 \%$, la moyenne étant de $25 \%$ (Fig.2). On peut en conclure qu'un quart au moins des interruptions, sont suivies d'une autre interruption. Cet indice peut être un indicateur de la disfluence dans un énoncé.

Figure 2. Proportions d'enchâssement des interruptions disfluentes chez les quatre locuteurs

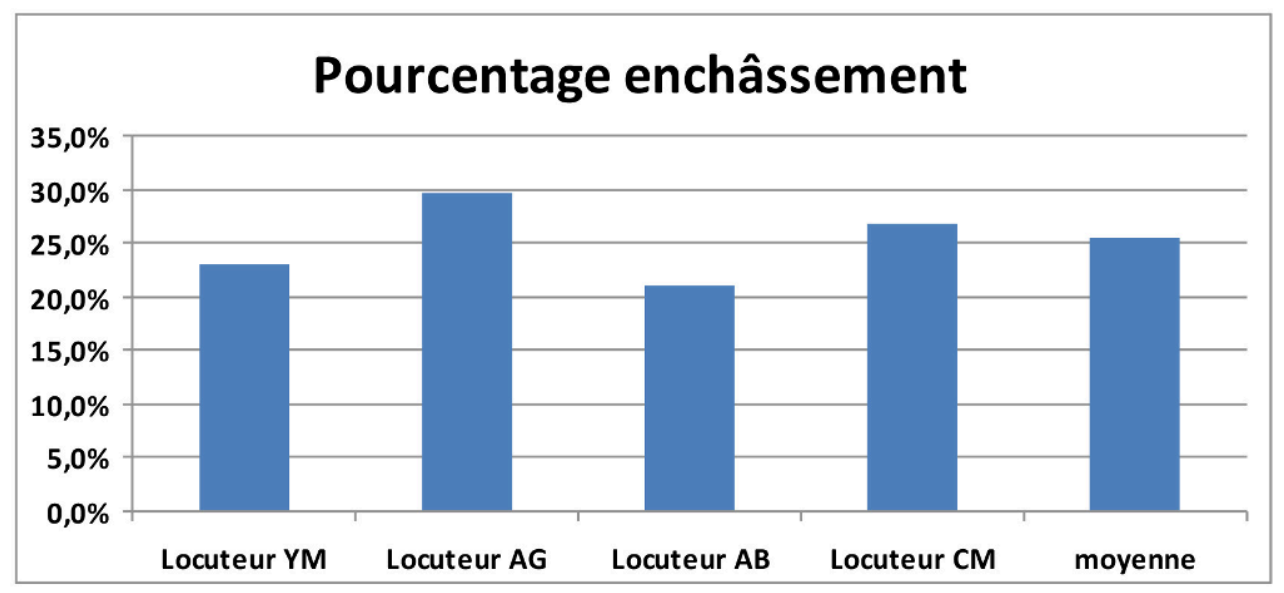

\subsection{Proportions des interruptions suspensives et disfluentes}

En moyenne, un peu plus de la moitié des interruptions observées (tableau 4) ne sont que suspensives, les autres étant disfluentes.

Ces deux conversations, l'une tenue entre deux hommes (locuteurs AG et YM), l'autre entre deux femmes (locutrices $\mathrm{AB}$ et $\mathrm{CM}$ ) diffèrent : les locutrices se caractérisent par des interruptions plus souvent suspensives que disfluentes alors que c'est l'inverse chez les locuteurs qui produisent plus de disfluences que de simples suspensions du flux 
verbal. Toutefois, cette observation, sur quatre locuteurs seulement, révèle avant tout une hétérogénéité entre les locuteurs.

Tableau 4. Parmi la totalité des interruptions, proportions d'interruptions suspensives et disfluentes chez les quatre locuteurs

\begin{tabular}{|l|l|l|}
\hline & Interruptions suspensives & Interruptions disfluentes \\
\hline Locuteur YM & $49,5 \%$ & $50,4 \%$ \\
\hline Locuteur AG & $48,1 \%$ & $51,9 \%$ \\
\hline Locuteur AB & $62,8 \%$ & $36,8 \%$ \\
\hline Locuteur CM & $50,8 \%$ & $49,0 \%$ \\
\hline Moyenne & $51,6 \%$ & $48,2 \%$ \\
\hline
\end{tabular}

\subsection{Les disfluences avec reprise d'un énoncé et les disfluences avec énoncés laissés inachevés}

On observe deux catégories d'interruptions disfluentes :

*celles qui provoquent la reprise d'une partie de l'énoncé interrompu avec une éventuelle modification de l'énoncé repris :

CM 884 mais les euh les nanas du foyer elles étaient pas au courant CM 996 tu as des des feux d'artifices comme ça

*celles qui, l'énoncé ayant été laissé inachevé, sont suivies d'une nouvelle construction ou d'un nouveau syntagme

CM 1139 Ah si mais j'ai // c'est un truc qui m'avait fait bien rire CM 1198 et je mes fixations sont pas bien réglées

Quel que soit le locuteur, parmi la totalité des interruptions, celles qui sont suivies d'une reprise disfluente sont largement plus nombreuses que celles où l'énoncé est laissé inachevé (en moyenne $\mathbf{3 0}$ \% versus $\mathbf{1 8} \%$; Fig 3). Le reste des interruptions n'est qu'une simple suspension du flux verbal (52\%) ${ }^{10}$. Que les disfluences avec reprise d'une partie de l'énoncé soient en nombre supérieur à celui des abandons d'énoncé a été observé également lors d'entretiens semi-directifs enregistrés (Pallaud \& Henry, 2007 ; Dister 2007). 
Figure 3. Types d'auto-interruptions dans les énoncés (abandons, simples poursuites ou reprises)

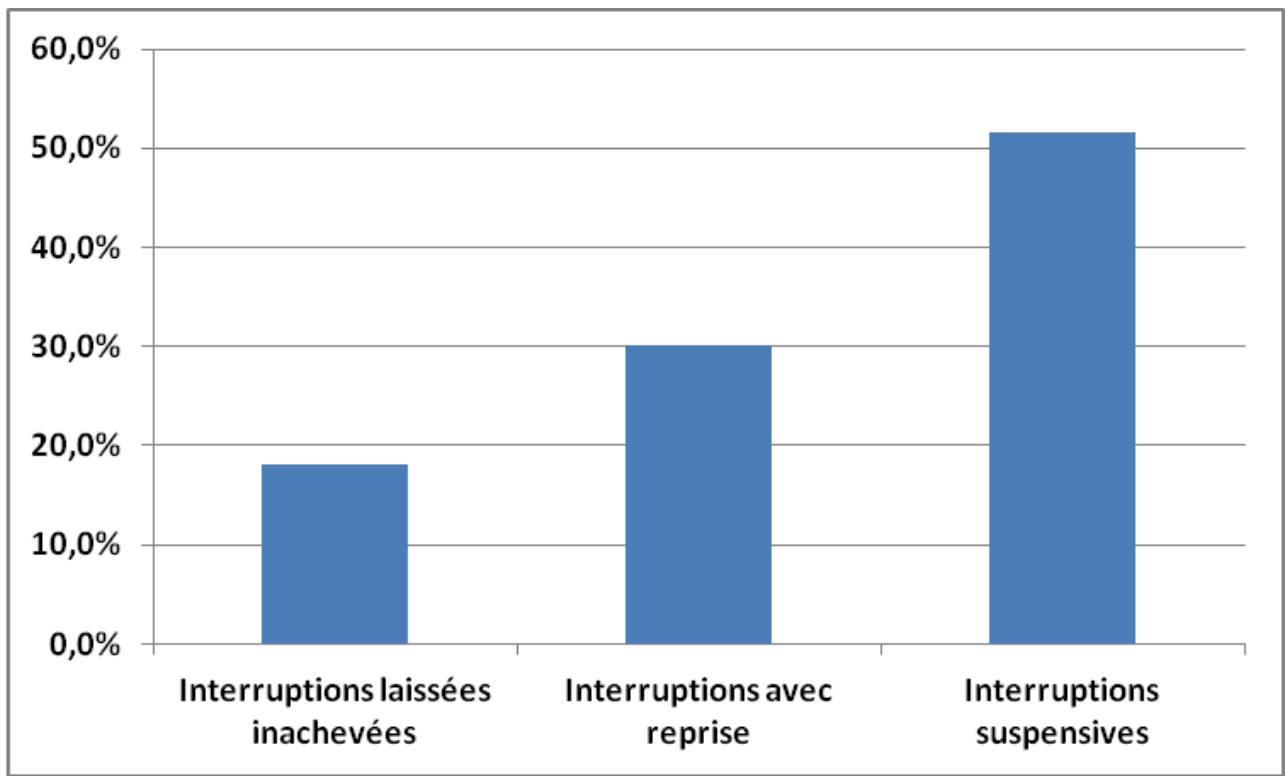

La variabilité entre interlocuteurs peut être importante (Tableau 5): les interruptions laissées inachevées varient de 10 à $20 \%$ et les interruptions avec reprise de 26 à $30 \%$. Toutefois less premières restent moins fréquentes que les secondes.

Tableau 5. Parmi la totalité des interruptions : proportions de disfluences avec reprises et laissées inachevées

\begin{tabular}{|l|l|l|}
\hline & Interruptions laissées inachevées & Interruptions avec reprise \\
\hline Locuteur YM & $19,9 \%$ & $30,5 \%$ \\
\hline Locuteur AG & $18,3 \%$ & $33,5 \%$ \\
\hline Locuteur AB & $10,5 \%$ & $26,3 \%$ \\
\hline Locuteur CM & $20,3 \%$ & $28,7 \%$ \\
\hline Moyenne & $18,0 \%$ & $30,2 \%$ \\
\hline
\end{tabular}

\subsection{Les indicateurs d'auto-interruption}

Cette étude analyse également les relations existant entre les interruptions suspensives ou disfluentes et la présence d'un indice d'interruption.

La collecte des interruptions présentes dans les énoncés des quatre locuteurs s'appuie sur deux méthodes : la détection des espaces Interregnum et, lorsqu'ils sont absents, les effets même de ces interruptions :

*Les espaces Interregnum sont de natures différentes et correspondent à des interruptions dans le flux verbal d'une durée variable. Nous en avons distingué quatre sortes: pauses silencieuses (d'une durée supérieure ou égale à 200ms) ou pauses 
remplies (euh), marqueurs discursifs. Ces éléments peuvent éventuellement être associés entre eux ; ces espaces Interregnum sont dits multiples.

*Par ailleurs, les discordances syntaxiques et/ou sémantiques révèlent des interruptions auxquelles ne correspond aucun espace interruptif observable. Cet espace Interregnum est alors dit vide.

Nos résultats ont montré (Fig. 3) que la moitié seulement des interruptions sont suivies d'une reprise ou d'un abandon de l'énoncé interrompu (et s'accompagne donc de disfluences). L'autre moitié n'est que suspensive puisque l'énoncé interrompu est simplement poursuivi sans reprise. Pour étudier si les indicateurs sont de même type et dans les mêmes proportions dans l'un et l'autre cas, nous avons suivi une méthode élaborée à partir des données étiquetées sous Praat puis transférées dans le tableur Excel.

\subsection{Répartition des indicateurs dans les deux cas d'interruption (disfluentes ou suspensives)}

49 Notre objectif est de répondre aux deux questions suivantes : comment les locuteurs interrompent-ils leurs énoncés? Cela diffère-t-il selon le type d'interruption (disfluente ou suspensive)?

50 Sur la totalité des interruptions observées, la catégorie des espaces vides représente $19 \%$ des cas. Cependant, elle est réservée aux interruptions disfluentes puisque l'interruption suspensive ne peut être détectée sans l'apparition d'un espace Interregnum rempli. Parmi les interruptions disfluentes, la proportion d'espaces Interregnum vide est donc très importante : elle représente $39 \%$ des cas de disfluence (en moyenne 200 cas par locuteur). Donc, un peu moins de la moitié des disfluences ne peut être détectée par notre méthode semi-automatique et a nécessité une méthode «manuelle » fondée sur la détection de perturbations morphosyntaxiques.

51 La comparaison des résultats obtenus pour les disfluences avec les interruptions suspensives (Fig.4) montre que si tous les types d'espace Interregnum remplis sont présents dans ces deux catégories d'interruptions, ils n'y sont pas dans les mêmes proportions.

52 Pour les interruptions disfluentes, la catégorie des espaces multiples, dans lesquels on trouve au moins deux éléments, est la plus fournie $(25 \%)$. Viennent ensuite les pauses silencieuses seules ( $17 \%$ des espaces succédant aux disfluences). Les espaces à un seul élément (connecteurs de discours et pauses remplies) constituent chacun $9 \%$ et $10 \%$ des Interregnum. 
Figure 4. Le contenu de l'espace Interregnum après les interruptions disfluentes et suspensives (les quatre locuteurs)

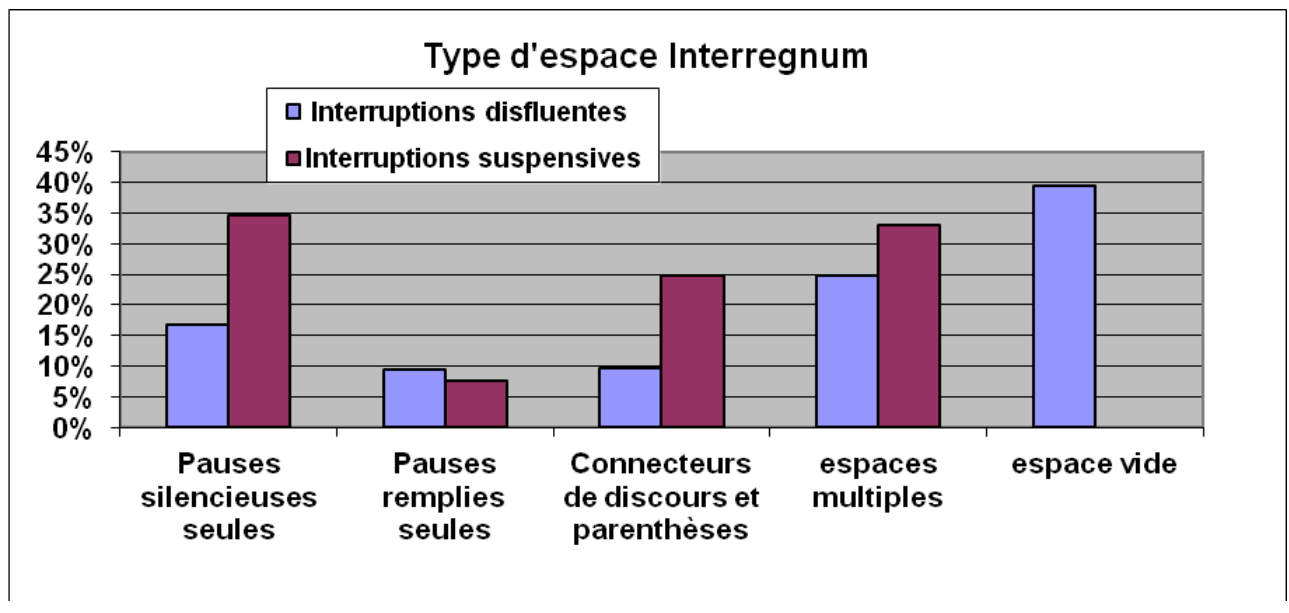

L'interruption suspensive, quant à elle, est suivie majoritairement par une pause silencieuse seule ( $35 \%)$ ou un espace multiple ( $33 \%$ des cas). Viennent ensuite les connecteurs de discours seuls $(25 \%)$ et les pauses remplies seules ( $8 \%)$.

Il semble que ce soit principalement sur les pauses silencieuses seules et les connecteurs de discours seuls que les interruptions suspensives diffèrent le plus des disfluences L'interruption suivie d'une pause silencieuse seule ou d'un connecteur de discours seul a beaucoup plus de chance d'être suspensive. Ces types d'espaces Interregnum y sont beaucoup plus fréquents. La pause remplie seule semble liée aussi bien à une simple suspension de l'énoncé qu'à une interruption disfluente.

55 Ainsi, comme on le constate, la pause silencieuse n'est pas réservée à l'interruption suspensive. Dans un certain nombre de cas (en moyenne 85 cas sur 274 dans notre étude), elle est associée à une interruption disfluente (Duez, 2001).

\subsection{Répartition des indicateurs dans les deux cas d'interruption disfluentes (avec reprise ou inachèvement)}

56 Les deux types de disfluence, interruptions laissées inachevées et interruptions avec reprise diffèrent également entre elles quant à leurs espaces Interregnum.

Nous avons vu (fig. 3) que, quel que soit le locuteur et quel que soit le type d'item interrompu, les disfluences avec reprise, comparées à la totalité des interruptions, sont largement plus nombreuses que celles où l'énoncé est laissé inachevé ( $30 \%$ versus $18 \%$ par rapport à la totalité des interruptions), (62\% versus $38 \%$ par rapport aux interruptions disfluentes). Ces résultats, obtenus dans des situations d'interaction, corroborent ce qui avait été observé lors d'entretiens semi-directifs où la prise de parole du locuteur interviewé est proche du monologue (Pallaud \& Henry, 2007). Le pourcentage de disfluences se traduisant par un abandon de l'énoncé (effet d'ellipse) par rapport aux cas avec reprise est supérieur dans le cas de ces quatre dilogues (38\%) à celui qui avait été observé dans le cas d'entretiens semi-directifs (25\%). De plus, cela reste vrai malgré une variation entre interlocuteurs importante. Le taux d'abandons d'énoncé ne dépasse pas les $42 \%$, et n'est pas inférieur à $29 \%$. 
Tableau 6. Disfluences avec reprise et inachèvement d'énoncés chez les quatre locuteurs

\begin{tabular}{|l|l|l|}
\hline & avec reprise & Inachevées \\
\hline Locuteur YM & $60 \%$ & $40 \%$ \\
\hline Locuteur AG & $67 \%$ & $33 \%$ \\
\hline Locuteur AB & $71 \%$ & $29 \%$ \\
\hline Locuteur CM & $58 \%$ & $42 \%$ \\
\hline Moyenne & $\mathbf{6 2 \%}$ & $38 \%$ \\
\hline
\end{tabular}
multiples et de pauses silencieuses seules ( $20 \%$ et $16 \%$ ), enfin, dans une moindre mesure, de connecteurs discursifs et de pauses remplies seules (10\% et $7 \%$ ). Au total, $52 \%$ des cas d'interruption avec reprise sont suivis d'un espace Interregnum rempli.

61 Ce n'est pas le cas des disfluences de type «inachevées » qui ont, de façon générale (dans trois quart des cas), des espaces interruptifs plus souvent remplis. Elles sont suivies dans un tiers des cas par des espaces multiples (33\%), par des pauses silencieuses seules et des connecteurs de discours (dans les mêmes proportions que les disfluences avec reprise (10\% et $15 \%$ ) ainsi que des pauses remplies (7\%).

En résumé, lorsque le locuteur interrompt son énoncé en le laissant inachevé, il poursuit son émission verbale en la précédant d'un moment «tampon » dans $75 \%$ des cas alors que s'il reprend une partie de son énoncé interrompu il n'utilise un moment «tampon» que dans la moitié des cas. La détection semi-automatique permet donc de détecter les trois quart des interruptions d'énoncés laissés inachevés et seulement la moitié des interruptions avec reprise.

63 Seules les proportions de marqueurs de discours ne diffèrent pas selon la catégorie de disfluence ; ils sont également présents dans les disfluences avec reprise et celles avec inachèvement (10\% dans les deux cas). 
Figure 5. Types d'insertions lors des interruptions disfluentes avec reprise (RA) et abandons de l'énoncé (I)

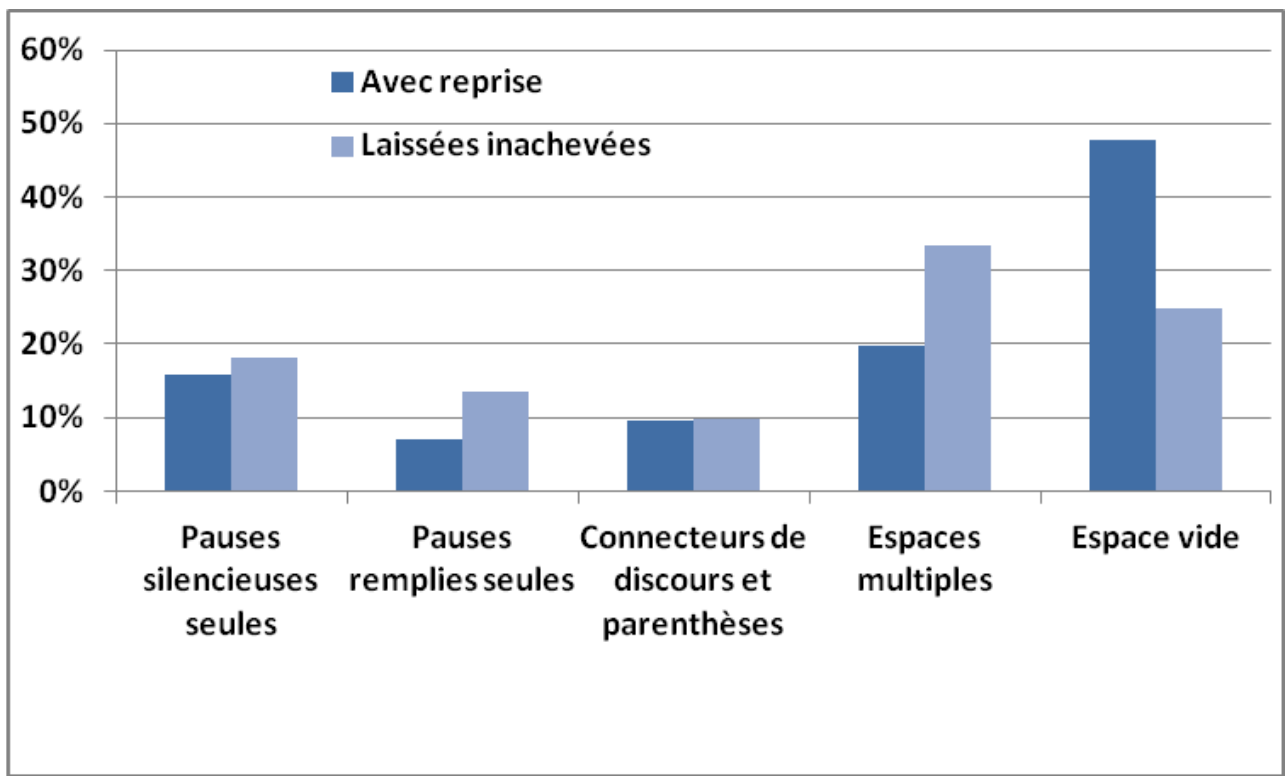

\subsection{Durée des phases d'auto-interruption et de disfluence dans les énoncés des quatre locuteurs}

Nous voulions déterminer l'importance de la disfluence morphosyntaxique pour chacun des locuteurs, ce qui demande de définir ce qu'est un énoncé « idéal » (c'est-àdire sans disfluences et sans pauses disfluentes). Evaluer la durée des prises de parole de chaque locuteur requiert d'une part de tenir compte des moments durant lesquels le locuteur partenaire parle (pauses interactives) et d'autre part de déterminer la durée des phases d'auto-interruption et particulièrement celle des phases de disfluence. Nous avons choisi de calculer la durée totale des pauses interactives pour chacun des locuteurs ce qui permet de déterminer la durée de l'énoncé total (chaque dilogue ne durant que 3572 secondes).

Elle nécessite d'annoter sur une Tier aux espaces de pauses (code \#) durant lesquels le locuteur partenaire parle et le locuteur observé se tait le plus souvent ${ }^{11}$. Le code employé pour annoter ces pauses interactives est « B,I ». Les intervalles durant lesquels le locuteur ne produit que des backchannels (mh, ouais, ah ouais) ne sont pas fractionnés en sous intervalles séparés par ces backchannels. Le transfert sous Excel de ces données ${ }^{12}$ permet de les analyser.

La durée de l'énoncé du locuteur est la durée totale du dilogue (3572 sec) diminuée de la durée des moments où le locuteur ne parle plus (pauses interactives; Tableau 7). La variation dans les durées maximum de ces pauses interactives indique que certains locuteurs ( $\mathrm{AB}$ et $\mathrm{CM}$ ) ont parlé sans discontinuer plus longuement que les locuteurs $\mathrm{YM}$ et AG. Elles sont nombreuses et durent en moyenne 7 secondes mais peuvent atteindre plus de 2 minutes (132 secondes). Ces conversations se caractérisent par de nombreux tours de parole. 
Tableau 7. Pauses interactives chez les quatre locuteurs

\begin{tabular}{|l|l|l|l|l|l|}
\hline $\begin{array}{l}\text { Pauses interactives } \\
\text { B,I (Durée en sec.) }\end{array}$ & $\begin{array}{l}\text { Durée } \\
\text { totale en } \\
\text { sec }\end{array}$ & $\begin{array}{l}\text { Nombre de pauses } \\
\text { interactives }\end{array}$ & $\begin{array}{l}\text { Durée } \\
\text { moyenne des } \\
\text { pauses }\end{array}$ & $\begin{array}{l}\text { Durée } \\
\text { minimum }\end{array}$ & $\begin{array}{l}\text { Durée } \\
\text { maximum }\end{array}$ \\
\hline Locuteur YM & 1445,05 & 230 & $\mathbf{6 , 2 8}$ & 0,20 & 52,62 \\
\hline Locuteur AG & 1645 & 253 & $\mathbf{6 , 5 0}$ & 0,20 & 49,64 \\
\hline Locuteur AB & 1665,82 & 219 & 7,61 & 0,18 & 97,87 \\
\hline Locuteur CM & 1655,28 & 217 & 7,63 & 0,18 & 131,75 \\
\hline
\end{tabular}

Les dilogues du CID ont été organisés afin de pouvoir analyser les énoncés de deux locuteurs au cours de leur conversation. Ce type d'interaction se caractérise par des successions de prise de parole se chevauchant parfois. En moyenne, la durée de prise de parole totale des locuteurs est de $\mathbf{3 2 , 8} \mathbf{m i n}$ (Tableau 9). Elle est variable d'un locuteur à l'autre (de $32 \mathrm{~min}$. à $35 \mathrm{~min}$.) mais cette variation est faible dans ces deux dilogues.

Tableau 8. Durées des pauses interactives et des énoncés prononcés pour les quatre locuteurs

\begin{tabular}{|l|l|l|l|l|}
\hline Locuteur/Durées & Dilogue & Pauses interactives & Enoncé prononcé & Enoncé en min. \\
\hline Locuteur YM & 3572 & $\mathbf{1 4 4 5 , 0 5}$ & $\mathbf{2 1 2 6 , 9 5}$ & 35,4 \\
\hline Locuteur AG & 3572 & $\mathbf{1 6 4 5}$ & $\mathbf{1 9 2 7}$ & 32,1 \\
\hline Locuteur AB & 3572 & $\mathbf{1 6 6 5 , 8 2}$ & $\mathbf{1 9 0 6 , 1 8}$ & 31,7 \\
\hline Locuteur CM & 3572 & $\mathbf{1 6 5 5 , 2 8}$ & $\mathbf{1 9 1 6 , 7 2}$ & 31,95 \\
\hline
\end{tabular}

Les proportions de disfluences sont calculées à partir des durées des énoncés prononcés. L'énoncé prononcé correspond à la prise de parole par le locuteur y compris lorsqu'il parle en même temps que l'autre locuteur. Les pauses interactives (cf tableau 7) sont les moments où le locuteur ne parle pas mais écoute l'autre locuteur.

70 L'énoncé prononcé comporte donc plusieurs éléments observables :

Les espaces Interregnum inclus dans les disfluences et les interruptions suspensives Tous les mots prononcés par le locuteur en dehors des pauses interactives et des espaces Interregnum

71 Exemple : énoncé prononcé total

\begin{tabular}{|l|l|l|l|l|l|l|l|}
\hline Je vais & euh bon & je vais & oublier & euh & mon & co- & colis \\
\hline RDum & B disf & Rans & RDum2 & B disf & Rans2 & & \\
\hline & & & & & & RDum3 & Rans3 \\
\hline
\end{tabular}


72 L'évaluation de la disfluence est faite à partir de la totalité des Reparandum (RDum, RDum2, RDum3) et des pauses disfluentes, diminuée des Reparans Rans sans reprise ${ }^{13}$ (Rans2 dans l'exemple).

Lorsque l'analyse porte sur les durées, les disfluences où le locuteur laisse son syntagme ou son mot inachevés ne sont pas concernées : elles constituent, elles aussi, l'énoncé « informatif ». Il n'en est pas de même si on comptabilise les tokens disfluents. Dans l'exemple, ci-dessus il y a six tokens disfluents (je vais euh bon euh co-) et cinq tokens non disfluents (je vais oublier mon colis) : soit plus de la moitié de disfluence. Comptabiliser les tokens pour évaluer la part de disfluence dans un énoncé conduit, semble-t-il à surévaluer ce phénomène. C'est pourquoi, il nous a paru plus pertinent d'évaluer la disfluence à l'aide des durées des tokens (Tableau 9).

Tableau 9. Durées en minutes et pourcentages de disfluence chez les quatre locuteurs

\begin{tabular}{|l|c|c|c|c|c|c|c|}
\hline $\begin{array}{l}\text { Locuteur/Durées } \\
\text { en min }\end{array}$ & $\begin{array}{c}\text { Enoncé } \\
\text { prononcé }\end{array}$ & $\begin{array}{c}\text { Espaces } \\
\text { Interregnum } \\
\mathrm{B}\end{array}$ & $\begin{array}{c}\text { Espaces } \\
\text { Reparandum } \\
\mathrm{R}\end{array}$ & $\begin{array}{c}\text { Reparans } \\
\text { RA,nr,co }\end{array}$ & $\begin{array}{c}\text { Enoncé } \\
\text { idéal }\end{array}$ & $\begin{array}{c}\text { Evaluation } \\
\text { disfluence }\end{array}$ & $\begin{array}{c}\text { Proportion } \\
\text { de } \\
\text { disfluence }\end{array}$ \\
\hline Locuteur YM & 35,4 & 10,9 & 5,6 & 2,4 & 21,4 & 14,1 & $39,7 \%$ \\
\hline Locuteur AG & 32,1 & 9,8 & 5,5 & 2,5 & 19,2 & 12,9 & $40,1 \%$ \\
\hline Locuteur AB & 31,7 & 8,6 & 4,7 & 2,7 & 21,2 & 10,6 & $33,3 \%$ \\
\hline Locuteur CM & 31,95 & 10,4 & 5,1 & 2,9 & 19,4 & 12,6 & $39,3 \%$ \\
\hline Moyenne & 32,8 & 9,9 & 5,2 & 2,6 & 20,3 & 12,5 & $38,1 \%$ \\
\hline
\end{tabular}

74 La durée de l'énoncé total comporte la durée de la totalité des mots prononcés informatifs (énoncé informatif) et la durée des éléments disfluents (reprises et espaces Interregnum). Le pourcentage de disfluence ainsi défini varie d'un locuteur à l'autre : il est de $\mathbf{3 8} \%$ en moyenne. En conséquence, sur un énoncé prononcé de 33 minutes, seules 20 minutes seront informatives. Près de 13 minutes seront consacrées à ce qu'on pourrait appeler l'échafaudage (quelle qu'en soit la fonction, respiratoire, hésitation, correction, etc.) pour l'élaboration de l'énoncé.

\section{Conclusion}

75 La méthode d'identification et d'annotation qui a été développée a permis d'introduire une classification dans les différents phénomènes oraux relevant de l'auto-interruption et de la disfluence. Si l'identification automatique des points d'auto-interruption pourrait être encore améliorée, la méthode manuelle s'est avérée indispensable pour prétendre à une certaine exhaustivité, ce qui a requis un temps d'identification important. Le recours à une analyse morphosyntaxique automatique devrait améliorer l'efficacité de cette méthode.

76 Le codage des annotations est hiérarchisé, il n'est donc pas obligatoire, suivant les analyses visées, de coder également les informations contenues dans les trois axes de la structure. On peut, par exemple, se limiter à l'identification des trois axes (Reparandum, Interregnum et Reparans). On peut ne s'intéresser qu'aux informations contenues dans le Reparandum (lieu de l'interruption et type de mot affecté). Inversement, si le codage est complet, les requêtes peuvent porter sur n'importe quelle information codée et déterminer quels facteurs sont importants dans la disfluence.

TIPA. Travaux interdisciplinaires sur la parole et le langage, 29 | 2013 
77 L'intérêt d'un système d'annotation focalisé sur les interruptions du flux verbal par des pauses ou éléments discursifs (indices de disfluence) est, outre d'interroger la notion même de disfluence morphosyntaxique, de montrer que ces événements qui n'entrent pas dans la construction de l'énoncé sont présents (mais dans des proportions différentes) dans ces interruptions quelles qu'elles soient. Un peu plus de la moitié de ces interruptions ne sont pas suivies d'une reprise ou d'un inachèvement de syntagme ou de mot, mais d'une simple poursuite de l'énoncé.

78 Les interruptions et réorganisations ne semblent, donc, pas, dans la grande majorité des cas, contrevenir au déroulement de la séquence verbale mais plutôt imposer un rythme propre aux énoncés oraux. Il semble même que ce rythme particulier à l'oral crée, au contraire, les conditions d'une interaction optimale dans la mesure où, en provoquant une réorganisation de l'énoncé et en intercalant des moments de silence ou d'interjections ${ }^{14}$ (au sens étymologique) variées, il allège pour le récepteur la charge informationnelle de l'énoncé (Boula de Mareüil et al., 2005, McAllister, et al. 2001; Pallaud \& Henry, 2004, Shriberg, 1995). Même, les interruptions de mots suivies de pauses et de correction permettent au récepteur une amélioration dans une tâche de choix d'objets (Brennan, 2001 ; Fox Tree, 1995).

Il est préférable d'ôter au terme de disfluence toute connotation péjorative liée par ailleurs au terme de dysfluence lui-même réservé aux phénomènes observés dans certaines pathologies comme le bégaiement (Pallaud \& Xuereb, 2008) ou la maladie de Parkinson, par exemple. De plus, les données obtenues sur des locuteurs «standard" permettent une stratégie de comparaison avec les faits observés chez des malades et d'en préciser les caractéristiques dysfluentes.

\section{BIBLIOGRAPHIE}

Adda-Decker M., Habert B., Barras C., Adda G., Boula de Mareüil P., Paroubek P., (2003) A disfluency study for cleaning spontaneous speech automatic transcripts and improving speech language models. Proceedings of DISS'03, Disfluency in spontaneous speech Workshop, Göteberg University, Sweden.

Bear, J., Dowding, J. \& Shriberg, E.E. (1992). Integrating multiple knowledge sources for detection and correction of repairs in human-computer dialog. Proc. Annual Meeting of the Association for Computational Linguistics, Newark, Delaware p. 56-63.

Bertrand R. Blache P, Espesser R. Ferre G., Meunier C., Priego-Valverde B. \& Rauzy S., (2009). Le CID- Corpus of Interactional Data- Annotation et exploitation multimodale de Parole conversationnelle.

Bigi, B; Bertrand, R; Guardiola, M (2010). Recherche automatique d'hétéro-répétitions dans un dialogue oral spontané. Actes, XVIIIèmes Journées d'Étude sur la Parole (2010 mai 24-28 : Mons, BELGIUM). 2010, p. 1-4. Cederom.

Blache P., Bertrand, R., Guardiola M., Guénot M.L., Meunier C., Nesterenko I, Pallaud B., Prévot L., Priego-Valverde B., Rauzy S., (2010) The OTIM formal annotation model: a preliminary step 
before annotation scheme. In Proceedings of LREC: Workshop on Multimodal Corpora, Valetta, Malta, p. 3262-3267.

Blanche-Benveniste C., (1997), Approches de la langue parlée en français. Paris, Edition Ophrys.

Boula de Mareüil P, Habert B., Bénard F., Adda-Decker M., Barras C., Adda G., Paroubek P. (2005), "A quantitative study of disfluencies in French broadcast interviews », ISCA TR Workshop on Disfluency in Spontaneous Speech (DISS), Aix-en-Provence, p. 27-32.

Brennan S.E.,(2001), How do listeners compensate for disfluencies in spontaneous speech. Journal of Memory and Language, 44, p. 274-296.

Clark H.H. et Wasow T., (1998), Repeating words in spontaneous speech, Cognitive Psychology, 37, p. 201-242.

Cole, J., Mark Hasegawa-Johnson, Chilin Shih, Eun-Kyung Lee, Heejin Kim, H. Lu, Yoonsook Mo, Tae-Jin Yoon. (2005). Prosodic Parallelism as a Cue to Repetition and Hesitation Disfluency, Proceedings of DISS'05 (An ISCA Tutorial and Research Workshop), Aix-en-Provence, France, p. 53-58.

Constant M.et Dister A., (2010) Automatic detection of disfluencies in speech transcriptions. In Massimo Pettorino, Antonella Giannini, Isabella Chiari, Francesca M. Dovetto (Eds.). Spoken Communication. Cambridge Scholars Publishing, p. 259-272.

Cutler, A., (1981), The reliability of speech error data. In: Cutler, A., 1981, (Ed.) Slips of the tongue and language production, Amsterdam Mouton publishers.

Den, Y. (2001). Are word repetitions really intended by the speaker? In Proceedings of Disfluency In Spontaneous Speech (DISS'01), August 29-31, 2001, University of Edinburgh, Scotland, p. 25-28.

Dister A. (2007). De la transcription à l'étiquetage morphosyntaxique. Le cas de la banque de données textuelles orales VALIBEL. Université de Louvain, Thèse non publiée.

Dister A. (2008 a). La notation subjective de la pause constitue-t-elle un bon indice pour le découpage de corpus oraux?, In M. Constant, A. Dister, L. Emirkanian, S. Piron (Éds), Description linguistique pour le traitement automatique du français, Cahiers du Cental 5 (Louvain-la-Neuve, Presses universitaires de Louvain, p. 165-186.

Dister A. (2008 b) L'autocorrection immédiate en Français parlé. le cas des déterminants. In colloque Journées sur l'Analyse des Données Textuelles (JADT'08), p. 397-408.

Duez D., (2001 a), Signification des hésitations dans la production et la perception de la parole spontanée, Revue Parole, 17-18-19, p. 113-138.

Duez, D. (2001 b). Caractéristiques acoustiques et phonétiques des pauses remplies dans la conversation en français. Travaux interdisciplinaires du Laboratoire parole et langage d'Aix-enProvence (TIPA), 20, p. 31-48.

Duez D., (2007) Prosodie et rythme. In P. Auzou Les dysarthries. Marseille: SOLAL.

Fox Tree J., (1995), The effects of false starts and repetitions on the processing of subsequent words in spontaneous speech, Journal of Memory and Language, 34, p. 709-738.

Guénot M.L., (2005), Parsing de l'oral : traiter les disfluences. TALN, Dourdan, 6-10 juin 2005, http://aune.lpl.univ-aix.fr/ guenot/Papiers/2005_TALN-DISFLUENCES.pdf.

Henry S.et Pallaud B., (2003), Word fragments and repeats in spontaneous spoken French. In:Eklund R., Disfluency in Spontaneous Speech Workshop, Proceedings of DiSS'03, 5-8 September 2003, Göteborg University, Sweden, p. 77-80. 
Jeanjean C. (1984), « Les ratés c'est fa-fabuleux ». Etude syntaxique des amorces et des répétitions., LINX, 10, Syntaxe et discours, p. 171-177.

Levelt W.J.M., (1989), Speaking. From intention to articulation. Cambridge, MIT Press.

McAllister, J., Cato-Symonds, S., \& Johnson, B. (2001). Listeners'ERP responses to false starts and repetitions in spontaneous speech. In Proceedings of Disfluency In Spontaneous Speech (DISS'01) August 29-31, 2001, University of Edinburgh, Scotland p. 65-68.

Meteer M. (1995), Dysfluency stylebook for the Switchboard corpus.

Pallaud B., (2002), Les amorces de mots comme faits autonymiques en langage oral, Recherches Sur le Français Parlé, 17, p. 79-102.

Pallaud B.et Henry S., (2004), Amorces de mots et répétitions : des hésitations plus que des erreurs en français parlé. In Le poids des mots. Actes des 7èmes Journées Internationales d'Analyse statistique des Données Textuelles, Louvain-la-Neuve, 10-12 mars 2004. Louvain, PUL, vol 2, p. 848-858.

Pallaud B., (2005), The re-adjustment of word-fragments in spontaneous spoken French. Proceedings of DISS 05. Disfluency in Spontaneous Speech Workshop. 10-12 September 2005, Aix-enProvence, France, p. 145-149.

Pallaud B. (2006), Les amorces de mots et leur contexte droit en français parlé spontané. Revue Travaux Interdisciplinaires de Parole et Langage, 24, p. 117-138.

Pallaud B. (2006), Troncations de mots, reprises et interruption syntaxique en français parlé spontané. in JADT 2006, 8e Journées internationales d'Analyse statistique des Données Textuelles, 20-22 avril 2006, Besançon, p. 707-715.

Pallaud B. et Henry S. (2007), Les effets langagiers des amorces de mots et des répétitions dans les énoncés de français parlé spontané, In B. Vaxelaire, Sock R., G. Kleiber \& F Marsac, (Eds) Perturbations et réajustements. Langue et Langage, Publications de l'Université Marc Bloch Strasbourg 2, p. 131-141.

Pallaud B., (2008), Dans quelle mesure les amorces de mot sont-elles des ellipses ? in Pitavy J.C. (Ed.) Ellipse et effacement. Du schème de phrase aux règles discursives : enjeux du constituant inarticulé et de la place vide. Presses universitaires de Saint-Etienne (PUSE), p. 259-268.

Pallaud B. et Xuereb R., (2008), Les troncations de mots chez un locuteur bègue. Travaux Interdisciplinaires de Parole et Langage, 26, p. 93-113.

Pasdeloup V. (1992), A prosodic model for french text-to-speech synthesis : a psycholinguistic approach. In Bailly, G; Benoit, C; Sawallis, T R. (eds), Talking Machines. Theories, Models, And Designs, p. 335-348.

Richard E.et Doquet C. (dir.), (2012), Les représentations de l'oral chez Lagarce. Continuité, discontinuité, reprise, Academia (Réf.: 20056).

Shriberg, E.E. (1995). Acoustic properties of disfluent repetitions. Proc. International Congress of Phonetic Sciences, 4, Stockholm, Sweden p. 384-387.

Shriberg E.et Stolcke A., (1998). How Far Do Speakers Back Up In Repairs? A Quantitative Model. Proc. Intl. Conf. on Spoken Language Processing, Sydney, Australia vol. 5, p. 2183-2186.

Shriberg, E.E. (1999), Phonetic consequences of speech disfluency. In Proceedings of the 14th International Congress on Phonetic Science, San Francisco, p. 619-622. 
Shu Chuan Tseng, (1999), Grammar, prosody and speech disfluencies in spoken dialogues. Thesis Bielefeld.

Shu Chuan Tseng, (2005), Taxonomy of spontaneous speech phenomena in Mandarin conversation. http://www.corpus4u.org/forum/upload/forum/2005080805441239.pdf.

Schuller B, Eyben F, and Rigoll G, (2008), Static and Dynamic Modelling for the Recognition of Non-verbal Vocalisations in Conversational Speech. In: E. André et al. (Eds.), Berlin Heidelberg Springer-Verlag p. 99-110.

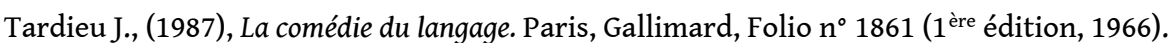

Van Riper C., (1971), Speech correction (5th ed.). New Jersey Prentice Hall.

Yoonsook Mo, Tae-Jin Yoon. (2005), Prosodic Parallelism as a Cue to Repetition and Hesitation Disfluency, Proceedings of DISS'05 (An ISCA Tutorial and Research Workshop), Aix-en-Provence, France, p. 53-58.

Zellner, B. (1992), Le bé- bégayage et euh ... l'hésitation en français spontané. Actes des 19èmes Journées d'Études sur la Parole, J.E.P. Bruxelles, p. 481-487.

\section{NOTES}

1. Le CID- Corpus of Interactional Data- Annotation et exploitation multimodale de Parole conversationnelle (2009).

2. Certains de ces phénomènes ont été transposés dans les œuvres théâtrales, en particulier par Tardieu (1966) et Lagarce (Richard et Doquet, 2012).

3. On ne se prononcera pas ici sur la question de savoir si ce sont les auto-interruptions et autovariations qui peuvent avoir des effets sur l'organisation morphosyntaxique du flux verbal, ou si ce sont les difficultés d'encodage morphosyntaxique qui peuvent avoir pour effet la présence d'auto-interruptions et d'auto-variations. (remarque de Frédéric Sabio; communication personnelle).

4. Le signe // représente le point d'interruption (IP selon la terminologie de Shriberg, 1999).

5. Nous avons repris la terminologie de Shriberg (1999) : Reparandum, Reparans, Interregnum.

6. L'absence d'indices d'interruption n'exclut pas néanmoins la présence d'indices acousticophonétiques (Shriberg, 1999). Ils ne sont pas analysés dans notre étude.

7. Dans le cas de ces quatre locuteurs, la liste est la suivante (23 éléments sur les 34 détectés sur la totalité des énoncés du CID ; communication personnelle de S. Rauzy) : ah, ben, bof, bon, bref, d'accord, eh, enfin, euh, hein, j'en sais rien, je sais pas, là, oh, non, ouais, oui, putain, quoi, si tu veux, tu vois, tu sais, voilà.

8. Les incises parenthétiques, si elles ne sont pas associées aux éléments cités plus haut, ne sont pas décelables automatiquement. Elles sont peu nombreuses au regard du nombre d'interruptions (7 cas sur $1381 \mathrm{chez}$ YM ; 17 sur 1130 pour AG ; 8 sur $695 \mathrm{chez}$ $\mathrm{AB}$ et 3 sur $1033 \mathrm{chez} \mathrm{CM}$ ); les autres sont accompagnées d'autres éléments, pauses silencieuses ou remplies, éléments discursifs et de ce fait détectables automatiquement.

9. A noter qu'il n'y a jamais de simple complétude d'un mot tronqué chez un locuteur standard. Un mot tronqué involontairement n'est complété qu'après une reprise au moins du début du mot (parfois du déterminant également; Pallaud., 2005, 2006 et 2006). En revanche, des personnes bègues peuvent compléter sans reprise un mot tronqué (Pallaud \& Xuereb, 2008). 
10. A noter que, dans les entretiens semi-directifs, les amorces de mots laissées inachevées sont une fois sur deux et à quelques exceptions près, les seules suivies d'une rupture de construction verbale (Pallaud, 2006).

11. Les locuteurs parlent aussi en même temps en plusieurs occasions dont la durée varie selon les locuteurs.

12. Le logiciel Praat a une fonction (List) qui fournit une liste des annotations sur trois colonnes (temps de début, annotation, temps de fin) pour chaque Tier.

13. Les Reparandum suivis d'un Reparans «simple poursuite» ne sont pas des disfluences. Ils sont évalués à partir de la durée de leurs Reparans.

14. Le Trésor de la Langue Française Informatisé signale son emprunt au latin. interjectio « action d'intercaler ».

\section{RÉSUMÉS}

Les énoncés oraux lors de conversations se caractérisent par de nombreuses variations dans le rythme de la fluence verbale et par des perturbations morphosyntaxiques. Toutes les autointerruptions et leurs effets ont été intégralement annotés dans quatre dilogues du CID. Cette étude en distinguant l'auto-interruption, ce qui la signale et les effets qu'elle provoque a permis d'analyser les relations existant entre ces événements. Le déroulement syntagmatique interrompu n'est pas toujours rompu: la moitié de ces ruptures ne sont que suspensives, les autres étant disfluentes. Tous les types d'insertion (espace Interregnum) sont présents dans l'un et l'autre cas d'interruption mais dans des proportions différentes. Le phénomène de reprise d'énoncé (par rapport à un abandon) après une auto-interruption disfluente semble être une caractéristique dominante des énoncés oraux. L'espace Interregnum qui lui succède n'est pas non plus le même. Le pourcentage de disfluences (durée des indices d'auto-interruption et des phénomènes de disfluence) par rapport à la durée du contenu informatif des émissions orales varie d'un locuteur à l'autre mais n'est pas inférieur au tiers de la durée de prise de parole par le locuteur.

Several phenomena of disfluency can be observed in the utterances of spontaneous oral conversation. They involve the phonetic, acoustic and prosodic levels as well as the morphosyntactic level. This study focuses on the morpho-syntactic level. Variations in the rhythm of verbal fluency (Pasdeloup, 1992; Duez, 2007; Béchet et al. 2013) can extend to the selfinterruption of words or phrases These self-breaks and auto-variations in the verbal fluency are related, in most of the cases, with one or several kinds of events or items inserted in the middle of a phrase or even a word. They can cause disturbances in the morpho-syntactic organization of verbal flow, the most frequently quoted being the resumptions after a break, such as auto-repairs and incomplete phrases or words (Clark \& Wasow, 1998; Henry \& Pallaud, 2003; 2007; Guenot, 2005; Pallaud \& Henry, 2006b).

Rather than trying to quantify and analyze some phenomena of disfluency previously defined (such as incomplete words or repetitions, for example), the preferred option was to identify all the auto-breaks in the statements and to describe the morpho-syntactic consequences of these interruptions in verbal flow. This approach, contrary to some previous work (for example Constant \& Dister, 2010), led us to distinguish what a self-interruption index is, i.e., the frequent signs of auto-breaks (silent or filled pauses, discursive markers, for example), and the effects of 
these ruptures on verbal flow. This made it possible on the one hand to exhaustively identify all the auto-breaks in a statement and on the other hand to describe the relations existing between these auto-breaks, their signs and their morpho-syntatic effects, which results in better specifying the concept of disfluency.

In order to describe all of the breaks in the statements of four corpora of the CID (Corpus of Interactional Data) and all of the morph-osyntactic disturbances, we successively used two methods of detection, one semi-automatic (detection of all Interregnum spaces; Shriberg 1994) and the other, a manual one. Both are used within the framework of the Praat software as a tool for identifying, describing and annotating the phenomena (Blache et al. 2010). The automatic method made it possible to identify only $81 \%$ of the breaks, the $19 \%$ remainder, were manually identified as being disfluent.

Main results

The breaks, thus stated, are numerous. On average, it is possible to find one rupture in the syntagmatic flow every 7.4 words (from 6.2 to 9.8 words, depending on the speakers). However, when the syntagmatic flow is stopped, it is not always broken: half of these ruptures are just hung up i.e. the statement is going on as if it had not been suspended. The other half causes a morpho-syntatic disturbance (unfinished or resumed statements); also their frequency strongly varies from one speaker to another: on average, it is one every 15.9 words.

Therefore, half of the detected breaks are on average, just suspending ones; the other half are disfluent. Whoever the speaker may be, among all the disfluent breaks, those which are followed by a resumption of the statement are far more numerous ( $62 \%$ versus $38 \%$ ) than those where the statement is left unfinished (results already obtained during recorded semi-directed talks (Pallaud \& Henry, 2007). The resumption of the statement after a disfluent auto-break seems thus to be a main characteristic in the oral statements.

This study equally analyzes the relationship existing between the suspending or disfluent breaks and the presence of a sign of interruption. If, in $81 \%$ of the cases only, the auto-interruptions, whatever they are, are announced by signs (silent or filled pauses, discursive interjections, markers), the others (19\%) were detected only by reading/listening which allows for the identification of resumptions or abandoning of a phrase or a word as they are not related to signs. This last category of breaks is by definition disfluent since, no signs being related to it, the disturbance of the statement (resuming or rupture patterns) is the only phenomena that makes it possible to detect these disfluencies. This fact has implications for the development of methods for automatic control of disfluent breaks.

All the types of insertion occur in both categories of breaks (suspendeding or disfluent ones) but in different proportions. We distinguished, in the Interregnum spaces, the silent or filled pauses alone, the speech markers alone and multiple spaces (containing more than one of the previous elements). The indicators related to the auto-break are not the same if the break is just suspending or if it is disfluent. When the Interregnum space contains a silent pause alone or a speech marker alone, in two cases out of three, this space follows a simple suspending of the statement. Moreover, the category of the disfluent breaks is the only one where the Interregnum space can be empty; it is the case for $39 \%$ of these breaks (which, therefore, were not detected automatically).

In addition to their morpho-syntactic effects, the two kinds of disfluent interruptions are distinguished by the content of the Interregnum space which succeed them. Only a quarter of the disfluencies left unfinished are followed by a filled Interregnum space whereas half of the disfluencies followed by a resumption are. Thus, the speakers back up in their statement and restart immediately in half of the cases whereas that occurs only in one quarter of the cases of a phrase or clause left unfinished. More precisely, when the Interregnum space is filled, the silent pauses and the speech markers (each alone) are in an equivalent proportion in the case of a restart or of an abandoning. It is not the same when the Interregnum space is filled with filled

TIPA. Travaux interdisciplinaires sur la parole et le langage, 29 | 2013 
pauses or multiple spaces. The disfluencies left unfinished are characterized by filled pauses alone or multiple spaces two times more frequent than in the disfluencies followed by a resumption,

The dialogs in the CID were organized in order to allow the analysis of the statements uttered by the two speakers during their conversation, which lasted one hour. This kind of interaction is characterized by a succession of talks that may overlap from time to time. On average, the duration of the whole talk for each speaker is $33 \mathrm{~min}$ and varies little according to the speakers (from 31.7 to $35 ; 4 \mathrm{~min}$ ). The evaluation of this duration comprises the duration of all the informative utterances (ideal statement) and the duration of the disfluent elements (Interregnum spaces and "repaired" items). We found that the percentage of thus defined disfluencies compared to the contents of the oral emissions varies from one speaker to another: it is $38 \%$ on average $(33,3 \%<\mathrm{X}<40,1 \%)$. As Bear and al. (1992) mentioned, "In summary, disfluencies occur at high enough rates in human-computer dialog to merit consideration." And that is not limited to the human-machine dialogs. This corpus of oral spontaneous statements shows that conversations also contain a lot of interruptions and several sorts of disfluencies.

\section{INDEX}

Keywords : spoken french, variation, auto-breaks, disfluency, morphosyntax

Mots-clés : français parlé, variation, auto-interruption, disfluence, morphosyntaxe

\section{AUTEURS}

\section{BERTHILLE PALLAUD}

Aix-Marseille Université, CNRS, LPL UMR 7309, Aix-en-Provence, France

berthille.pallaud@orange.fr

\section{STÉPHANE RAUZY}

Aix-Marseille Université, CNRS, LPL UMR 7309, Aix-en-Provence, France stephane.rauzy@lpl-aix.fr

PHILIPPE BLACHE

Aix-Marseille Université, CNRS, LPL UMR 7309, Aix-en-Provence, France

philippe.blache@lpl-aix.fr 